\title{
Editorial
}

\section{Patrick Hunziker}

\section{Nanomedicine translation from enabling technologies to the patient: focus on infectious diseases}

DOI 10.1515/ejnm-2016-0015

Nanomedicine is the indispensable enabling technology for the medicine of the future, encompassing molecular precision diagnosis, in vivo molecular imaging and targeted individualized therapies leading to personalized medicine.

Infectious diseases, including "old" diseases like tuberculosis, malaria as well as more recent, but equally widespread threats like HIV infection and the anticipated Zika virus epidemia belong to the major threats for health and human well being and are considerable burdens for individuals and healthcare systems.

Nanomedicine has delivered and will further contribute new tools for preventing infections (e.g. through nano-structured surfaces inhibiting bacterial colonization), for diagnosing infections early [e.g. through micro/ nano-based rapid testing, microfluidic-based miniaturized immunoassays and novel nucleic acid testing strategies (1-4)], for treating such infections in a targeted fashion, with the goals to cure (5) such diseases in the short term in an individual and in the long term, to eradicate (6) such diseases entirely.

As the progress in experimental nanomedicine in the new millennium has been breath-taking at the technical level, it is now the prime time for broad translation of such academic achievements towards clinical application. In view of this long-term goal of individual and societal benefit through industrial and clinical translation, it is of critical importance to identify translation roadblocks (7) and to find optimal matches between biomedical requirements, technical solutions, manufacturing issues (8), regulatory paths, and in particular, to choose the clinical trials strategies wisely, as this aspect will determine the acceptance of new solutions by the medical community.

A new breed of nano scientists and in particular nanomedical scientists is arising from its roots that range from experimental physics to pharmaceutics, from chemistry to biology, thereby radically changing the composition of academic research groups towards broad interdisciplinarity. However, even more interdisciplinarity is needed: Important fields of science are not yet well represented in nanomedicine, although it is felt that they might contribute significantly to this field. For example, theoretical physics, computational science (9) and physical chemistry might contribute significant aspects to deeper fundamental understanding, e.g. about the relevance of quantum effects in nanomaterials which may be much more important than we realize now. In a very different manner, the science of economics is urgently needed to delineate paths towards individually and societally affordable strategies for putting personalized medicine into practice. Philosophical, ethical fundaments and social factors that have a critical impact on translation and that will play a major role in the practice of medicine based on novel technologies will need thorough analysis.

Nanomedicine, which is probably already now "the most interdisciplinary of all sciences", will further profit from such a very broad vision of science, technology, medicine and society. This journal, and the related CLINAM conferences, will therefore continue to challenge scientists, physicians, industrial specialists, ethicists, regulators and government players, investors and all other stakeholders to contribute to such a comprehensive approach to medicine.

Such concerted efforts are needed for tackling the major challenges ahead in medicine, and in particular to overcome specific infectious diseases. For delivering a benefit to individuals and to mankind in rich and poor countries alike, such efforts are needed, are challenging, and will be highly rewarding not only to individuals and the healthcare systems benefiting from it, but also for those involved in making the future a reality.

\section{References}

1. Zhang B, Salieb-Beugelaar GB, Nigo MM, Weidmann M, Hunziker $P$. Diagnosing dengue virus infection - rapid tests and the role of micro/nanotechnologies. Nanomedicine 2015;11:1745-61. 
2. Zimmermann M, Hunziker P, Delamarche E. Autonomous capillary system for one-step immunoassays. Biomed Microdevices 2009;11:1-8.

3. Salieb-Beugelaar GB, Hunziker PR. Towards nano-diagnostics for rapid diagnosis of infectious diseases-current technological state. Eur J Nanomed 2014;6:11-28.

4. Salieb-Beugelaar GB, Zhang B, Nigo MM, Frischmann S, Hunziker PR. Improving diagnosis of pneumococcal disease by multiparameter testing and micro/nanotechnologies. Eur J Nanomed 2016;8:115-28.

5. Hunziker P. Toward personalized and curative medicine. In: Fadeel B, editor. Handbook of safety assessment of nanomaterials: from toxicological testing. Singapore: Pan Standford Publishing 2014:523-32; Print ISBN: 9789814463362 eBook ISBN.

6. Gonçalves D, Hunziker P. Transmission-blocking strategies: the roadmap from laboratory bench to the community. Malar J 2016;15:95.
7. Satalkar P, Elger BS, Hunziker P, Shaw D. Challenges of clinical translation in nanomedicine: a qualitative study. Nanomedicine 2016;12:893-900.

8. Liu K, Zhu Z, Wang X, Gonçalves D, Zhang B, Hierlemann A, et al. Microfluidics-based single-step preparation of injection-ready polymeric nanosystems for medical imaging and drug delivery. Nanoscale 2015;7:16983-93.

9. Hunziker P. Nanomedicine enabled by computational sciences. Eur J Nanomed 2013;5:173-4.

\section{Patrick Hunziker}

University Hospital Basel, Clinic for Intensive Care, Basel, Switzerland,

E-mail: Patrick.Hunziker@swissnano.org 